\title{
Screening for abdominal aortic aneurysms in Canada: 2020 review and position statement of the Canadian Society for Vascular Surgery
}

\author{
Varun Kapila, MD \\ Prasad Jetty, MD, MSc \\ Doug Wooster, MD \\ Vic Vucemilo, MD \\ Luc Dubois, MD, MSc; \\ on behalf of the Canadian \\ Society for Vascular Surgery \\ Accepted Sept. 18, 2020 \\ Correspondence to: \\ V. Kapila \\ Canadian Society for Vascular Surgery \\ PO Box 58062 \\ Ottawa ON K1C 7H4 \\ varun.kapila@utoronto.ca
}

Cite as: Can J Surg 2021 September 1; 64(5). doi: 10.1503/cjs.009120

\begin{abstract}
Abdominal aortic aneurysms (AAAs) remain a major risk to patients, despite level 1 evidence for screening to prevent rupture events and decrease mortality. In 2007, the Canadian Society for Vascular Surgery (CSVS) published a review and position statement for AAA screening in Canada. Since that publication, there have been a number of updates in the published literature affecting screening recommendations. In this paper, we present a review of some of the controversies in the AAA screening literature to help elucidate differences in the various published screening guidelines. This article represents a review of the data and updated recommendations for AAA screening in the Canadian population on behalf of the CSVS.
\end{abstract}

Les anévrismes de l'aorte abdominale (AAA) continuent de poser un risque majeur pour les patients, malgré des données probantes de niveau 1 à l'appui du dépistage pour prévenir les ruptures et réduire la mortalité. En 2007, la Société canadienne de chirurgie vasculaire (SCCV) a publié une revue et un énoncé de position sur le dépistage de l'AAA au Canada. Depuis lors, plusieurs mises à jour ont paru dans la littérature et elles ont un impact sur les recommandations relatives au dépistage. Dans le présent article, nous présentons une synthèse de quelques controverses soulevées dans la littérature sur le dépistage de l'AAA afin d'expliquer les différences entre les diverses lignes directrices publiées à ce sujet. Cet article propose au nom de la SCCV une revue des données probantes et des recommandations à jour sur le dépistage de l'AAA dans la population canadienne.

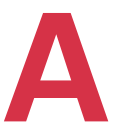
$\mathrm{n}$ abdominal aortic aneurysm (AAA) occurs when the abdominal aorta is more than $3 \mathrm{~cm}$ in maximal diameter, about 1.5 times larger than normal. The development of AAAs is associated with traditional cardiovascular risk factors, such as smoking, age, male sex, hypercholesterolemia and family history. ${ }^{1}$ The prevalence of AAAs in the general population aged 66 years or older ranges between $1 \%$ and $5 \%,{ }^{2}$ with a lower incidence in females, those with diabetes and those of African descent. ${ }^{3}$ As the aorta expands beyond its normal size, the risk of aortic rupture increases. Rupture is often fatal, with more than $80 \%$ of all patients with ruptured AAAs dying, many before hospital admission or even after emergent surgery. These aneurysms are often asymptomatic and therefore underdiagnosed, with symptoms occurring only once the aneurysm has ruptured or immediately before rupture.

Detecting AAAs before they pose a threat to life has proven to be an excellent way to decrease the devastating sequelae of untreated large aneurysms. Screening with abdominal ultrasonography has proven highly effective and can be performed in a variety of settings, with high specificity and sensitivity. ${ }^{4}$ Detecting AAAs before they rupture has been shown to reduce aorticspecific mortality, all-cause mortality and rupture events, while proving to be cost-effective. ${ }^{4}$

In 2007, the Canadian Society for Vascular Surgery (CSVS) published a review and position statement for AAA screening in Canada. ${ }^{5}$ Since that 
publication, there have been updates to the published literature affecting screening recommendations. The CSVS enlisted a panel to review the literature and produce consensus recommendations for AAA screening practices for CSVS members, primary care providers and patients.

\section{CURRENT STATE OF ABDominal AORTIC ANEURYSM SCREENING}

Despite the evidence supporting AAA screening, there are few structured national screening programs. The United Kingdom, Sweden and parts of Norway currently offer screening, with other countries currently evaluating the cost-effectiveness of screening programs before implementing them. ${ }^{6}$ In the United States, the Screening Abdominal Aortic Aneurysms Very Efficiently (SAAAVE) Act, effective as of Jan. 1, 2007, allows one-time free screening for patients at risk (men aged $>65 \mathrm{yr}$ who have ever smoked, and men and women with a family history of AAA disease) as part of the "Welcome to Medicare" physical examination. In Canada, currently no national or provincial screening program exists.

The CSVS's position statement on AAA screening included screening for all men aged 65-75 and for women aged 66 or older with history of smoking, family history of AAA or cerebrovascular disease. ${ }^{5}$ Since its publication, there have been updates from the 4 large population-based AAA screening studies, ${ }^{7-10} 2$ North American task force systematic reviews, ${ }^{11}$ a recent US Society for Vascular Surgery guidelines publication ${ }^{12}$ and other studies reporting real-life experience of national screening studies. ${ }^{13-15}$

In addition, in 2017, the Canadian Task Force on Preventive Health Care published recommendations for AAA screening in Canada that included one-time screening in men aged $65-80$ but no screening in women. ${ }^{16}$ The recommendations were a major departure from previous Canadian and other North American recommendations, especially regarding screening of women.

\section{Development of 2020 Canadian Society for VASCULAR SURGERY POSITION STATEMENT}

The position statement panel consisted of members from the CSVS with experience in the full spectrum of care and therapies of AAA management. These members were highly selected by the executive of the CSVS. Any potential members who had any conflict of interest or disclosure were excluded.

The panel members reviewed the data from the 4 major randomized controlled trials (RCTs) ${ }^{2,7,8,13}$ and the more recent publications describing their long-term followup. ${ }^{7-10}$ In addition, the panel reviewed systematic reviews and observational studies including results from large national screening programs. A review of all the various published screening recommendations was also performed.
The strength of the panel's recommendations and the quality of evidence was determined with the Grading of Recommendations Assessment, Development and Evaluation (GRADE) system. ${ }^{17}$ The quality of evidence from randomized trials has an initial rating of "high," which is further modified based on consideration of risk of bias, inconsistency of the results across studies, indirectness of the evidence, imprecision of the estimates of effect and study limitations. High-quality evidence that an intervention's desirable effects are clearly greater than its undesirable effects, or are clearly not, warrants a "strong" recommendation, whereas uncertainty about the trade-offs (because of low-quality evidence or because the desirable and undesirable effects are closely balanced) warrants a "weak" recommendation.

\section{CONTROVERSIES IN ABDOMINAL AORTIC ANEURYSM SCREENING}

From the detailed review of the literature, the panel identified controversies that have likely contributed to some of the disparities in screening recommendations for AAAs. ${ }^{5,12,16}$ By discussing these topics, the panel was ultimately able to provide more robust recommendations for screening.

\section{Decline in prevalence of abdominal aortic aneurysm at the population level}

The Multicentre Aneurysm Screening Study (MASS), an RCT of screening for AAA in a large UK population, showed a prevalence of an aortic aneurysm of $4.9 \% .^{2,9}$ This trial was considered the benchmark for screening recommendations as it showed a significant reduction in aorticspecific mortality and a modest reduction in overall mortality associated with ultrasonography screening.

These benefits have been called into question with the publication of more recent population-level studies, which have shown a much lower prevalence of AAA. In a nationwide Swedish AAA screening program offered to all men older than 65 years, the prevalence of AAA was $1.5 \% .{ }^{13}$ Similarly, a study from Gloucestershire showed a decrease in AAA prevalence from $5 \%$ to $1.3 \%$ over 25 years in their screening program. ${ }^{18}$ Among the first 700000 men screened (2009-2013) in the Abdominal Aortic Aneurysm Screening Programme in the UK, the prevalence of AAA was $1.3 \% .{ }^{14}$ A reduction in smoking rates and better modification of cardiovascular risk factors have been cited as reasons for the decline in AAA prevalence. ${ }^{19}$ This does result in having to screen a larger number of patients in order to prevent AAA-related events, which affects the potential cost-effectiveness of the screening program and its expected gains in reduced overall mortality as AAA-related deaths become less common. 


\section{Decline in mortality rates after abdominal aortic aneurysm repair}

With the development of endovascular techniques for AAA repair (EVAR) and better perioperative care for patients undergoing open surgical AAA repair (OSR), the risks of operative intervention have decreased, which may further increase the benefits of screening. In the MASS, the mortality rate after elective OSR was $6 \% .^{2}$ More recent studies of aneurysm screening programs that include a mix of elective EVAR and OSR have shown substantially lower rates of postoperative mortality. In the Swedish nationwide screening program, the mortality rate after elective aneurysm repair was $0.9 \%,{ }^{13}$ and in the UK Abdominal Aortic Aneurysm Screening Programme, the mortality rate was $0.8 \%$ in patients who had their aneurysm treated after it was detected by means of screening ultrasonography. ${ }^{14}$

If modern surgical therapy can be provided in a safer and more minimally invasive fashion, the benefits of screening and, ultimately, intervention may be greater than traditionally reported. This has helped to balance the riskbenefit ratio of AAA screening in the face of lower AAA prevalence. In a simulation study, Svensjö and colleagues ${ }^{20}$ estimated that AAA screening would still be cost-effective even with an AAA prevalence rate of only $0.5 \%$, given the improved postoperative outcomes seen with elective repair of aneurysms detected on screening.

\section{Uncertainties regarding risks and benefits of screening in women}

In most AAA screening studies, women were either excluded or underrepresented. Only 1 of the 4 major RCTs included women, ${ }^{10,15}$ and they accounted for only a small proportion (about $7 \%$ ) of the patients in that trial. In the US, almost $40 \%$ of all AAA-related deaths and onethird of hospital admissions for ruptured AAA occur in women. ${ }^{21,22}$ The UK Small Aneurysm Trial showed a rupture rate 3 times higher in women than in men, ${ }^{23}$ and a more recent study indicates that this excess risk may be as high as fourfold. ${ }^{4}$

The prevalence of AAA in women is relatively low, with one screening study indicating a prevalence of $1.3 \%$, compared with $7.6 \%$ for men. ${ }^{15}$ This lower prevalence is somewhat offset by the fact that AAAs in women tend to rupture more frequently and at lower aortic diameters. ${ }^{23}$ In addition, women have higher mortality rates after both elective and emergent aneurysm repair. ${ }^{25}$ Furthermore, the anatomy of AAAs in women is distinct, with a larger proportion not fulfilling the standard anatomic criteria for EVAR compared to those in men. ${ }^{26}$ The inconsistency between guidelines for AAA screening in women is understandable, as there is a paucity of evidence to guide recommendations. However, although AAA is less common in women, it is a much deadlier diagnosis than in men. Currently, national screening programs in Italy, New Zealand and the US include women. ${ }^{6}$

\section{RECOMMENDATIONS FOR ABDOMINAL AORTIC ANEURYSM SCREENING}

\section{Men aged $65-80$ years}

The 4 major screening trials ${ }^{2,7-10}$ showed a decrease in AAArelated mortality in men who were screened. Screening is also associated with a decreased risk of AAA rupture and emergency procedures, including a decrease in mortality after any AAA-related procedure. ${ }^{16}$ The number needed to screen to prevent an AAA-related death is estimated at 311, better than the benefit of breast cancer screening (number needed to screen $=1904^{27}$ ) or fecal occult blood testing for colon cancer (number needed to screen $=1374^{28}$ ).

Although the largest of the 4 major screening studies limited the upper age to 74 years, ${ }^{2}$ the Chichester ${ }^{10}$ and Western Australia ${ }^{29}$ studies had higher upper age limits (80 $\mathrm{yr}$ and $83 \mathrm{yr}$, respectively) and showed benefit in these more inclusive cohorts. Given the aging population and excellent results of both OSR and EVAR in the modern era, we believe including patients aged $65-80$ years will improve yield in AAA screening. Furthermore, a substantial proportion of patients in population-based aneurysm studies are within this inclusive age group at the time of aneurysm repair. ${ }^{13-15,18,30-32}$ It is also important to note that there may have been a selection bias in the trial data, as in the Western Australia study, ${ }^{29}$ in which those older than 75 were less likely than younger people to participate in AAA screening.

We recommend one-time screening ultrasonography for all men aged 65-80 years (grade 1a [strong, high-quality] evidence).

\section{Women aged $65-80$ years}

The issue of AAA screening in women is important, given the discrepant recommendations between previous and current guidelines. In the Chichester study, women aged $65-80$ were included. ${ }^{15}$ The study did not identify a benefit to screening women but was significantly underpowered to determine differences in this subgroup.

A US screening study that included 10012 women showed an overall prevalence of AAA of $0.7 \%$, but the prevalence was higher in certain risk factor groups. ${ }^{33}$ Women older than 65 with at least 1 risk factor for AAA were included. The prevalence of AAA among men was $3.9 \%$. A history of smoking or cardiovascular disease (previous myocardial infarction, coronary revascularization or other cardiac surgery), or increased age significantly increased the prevalence of AAA. The prevalence was 1.4\% among women aged $75-85$ and $2.7 \%$ among women older than age 85 . Among patients with a history of tobacco use or heart disease, the prevalence rose to $3.4 \%$, and the addition of family 
history increased the prevalence to $6.4 \%$. Women with a history of tobacco use or cardiovascular disease had a threeto fourfold increased prevalence with either risk factor.

The Women's Health Initiative Cohort Study assessed a myriad of cardiovascular events, including AAA events, in more than 160000 patients aged 50-79 over nearly 8 years. ${ }^{30}$ Abdominal aortic aneurysm events were strongly associated with smoking and increased age. Current smokers were at higher risk than patients who had ever smoked (odds ratio 4.19 v. 1.94).

Many national screening studies have shown costeffectiveness in screening for AAA in men, despite a prevalence of $1 \%-2 \% .{ }^{2,34}$ It follows that women with a similar disease prevalence and known poorer long-term outcomes of AAA should be similarly screened and that screening should be beneficial and cost-effective. In recent reports of AAA interventions, women accounted for about 20\%-25\% of AAAs treated. ${ }^{35,36} \mathrm{We}$ believe that the lack of screening data for women should not exclude this group from the possible benefits of ultrasonography screening.

We suggest one-time screening ultrasonography for all women aged 65-80 years with a history of smoking or cardiovascular disease (grade 2c [weak, low-quality] evidence).

\section{Men and women older than 80 years of age}

There is a paucity of data on screening patients older than 80 years. Many of the previous guidelines or recommendations were made before the widespread use of endovascular techniques. These minimally invasive therapies reduce procedural risks and should result in broader applicability of screening recommendations. As experience and advances in OSR and perioperative management improve, traditional open surgical therapies now carry less risk for older patients. Screening recommendations should also parallel real-world experience, and published aneurysm series include a substantial number of patients treated after age $80 . .^{31,32,37}$ In the IMPROVE trial, the average patient age was 76.7 years, ${ }^{31}$ and in a Canadian study of more than 1000 consecutive endovascular repairs, the average patient age was $79 .{ }^{32}$ Makrygiannis and colleagues ${ }^{37}$ studied a screening program in a cohort aged 75-85 and found an almost threefold increase in AAA prevalence in men and women compared to a younger cohort (65-74 yr).

Real-world experience with aneurysm repair in older adults has given acceptable results with high procedural success; women accounted for about $20 \%$ of patients. ${ }^{38,39} \mathrm{It}$ has been shown that women experience AAA-associated death at older ages, with $70 \%$ of deaths occurring after age $80 . .^{24,40}$ Selecting an arbitrary age cohort suited for AAA screening in men may not be sufficient to answer the question of benefit in women. With the increasing life expectancy of the North American population and the larger cohort of older patients with higher AAA prevalence, coupled with the excellent results of both OSR and EVAR in older adults, screening in older patients should be individualized based on life expectancy and patient choice.

We suggest consideration of AAA screening on an individual patient basis in men and women older than 80 years, depending on the patient's life expectancy and patient choice (grade 2c [weak, low-quality] evidence).

\section{First-degree relatives}

Although AAAs are thought of as a multifactorial disease, reports have consistently shown familial aggregation, with an incidence in first-degree relatives of the proband (index patient with the AAA) about 5-10 times that in the general population. ${ }^{41-45}$ One Canadian study showed a prevalence of AAA of $19.2 \%$ among siblings of 166 probands, compared to $2.3 \%$ in a control population. ${ }^{46}$ In particular, female first-degree relatives of probands have a high prevalence of AAA compared to all women in the general population ( $5 \%-7 \%$ v. $0.5 \%)$, although the prevalence of AAA is always higher in men regardless of the presence or absence of a proband. ${ }^{47,48}$

Aneurysms in first-degree relatives occur at an earlier age and may have a more pernicious natural history, with higher rates of rupture. ${ }^{44,47-50}$ As such, an improved benefit from targeted screening for AAA in proband-related populations has been suggested. ${ }^{12,51,52}$ In a recent study, Hultgren and colleagues ${ }^{53}$ performed model-based analysis and found that asking probands about their siblings and then inviting the siblings for screening could reduce mortality from AAA, with further improved costeffectiveness compared to AAA screening in the general population.

We suggest one-time screening ultrasonography after 55 years of age for all first-degree relatives of patients with AAA (grade 2c [weak, low-quality] evidence).

\section{Repeat screening for ectatic nonaneurysmal abdominal aortas}

Initial screening ultrasonography showing an ectatic aorta $(2-3 \mathrm{~cm})$ may not effectively identify all patients who may ultimately develop an AAA or, worse, a ruptured AAA. The ectatic aorta, although not aneurysmal, is abnormal, with the same degenerative events occurring within the aortic wall that may predispose to further degeneration. Typically, the aorta in patients with an aneurysm grows 1-3 mm per year, and most patients will die from other causes before the aorta reaches surgical maturity.

The MASS showed that, among the 25500 patients who had normal aortic diameters at the time of screening, 60 experienced a ruptured aneurysm, the majority of which had a diameter of $2.5-2.9 \mathrm{~cm} .{ }^{9}$ In a Swedish study following ectatic aortas measuring $2.5-2.9 \mathrm{~cm}$ in women, $46 \%$ had progressed to an AAA at 5 years. ${ }^{54}$ 
Although not well studied in the literature, it seems that larger ectatic aortas $(>2.5 \mathrm{~cm}$ but $<3 \mathrm{~cm}$ ) may require repeat assessment, especially in patients who have a long life expectancy and may decide on repair at an older age. Currently, no evidence exists as to the cost-effectiveness or aortic-specific benefits of this strategy. However, it appears that some patients with an ectatic aorta on initial screening may be falsely reassured regarding future aortic events and may benefit from rescreening. 9,54

We suggest consideration of repeat ultrasonography 10 years after the initial screening in patients with an initial aortic diameter greater than $2.5 \mathrm{~cm}$ and less than $3 \mathrm{~cm}$, depending on the patient's life expectancy and patient choice (grade 2c [weak, low-quality] evidence).

\section{Use of previous other imaging studies in lieu of screening ultrasonography}

In the modern era, in which the use of radiologic imaging continues to increase, the average person may have had imaging modalities other than ultrasonography for indications not related to AAA screening. If an imaging study has been performed in which the abdominal aorta can be seen in its entirety, with a report commenting on aortic measurement or the absence of an AAA, this would obviate the need for screening ultrasonography.

\section{Conclusion}

Abdominal aortic aneurysms are life-threatening conditions that can be easily detected with ultrasonography, which has been shown to save lives and prevent aortic rupture. This simple tool can be applied to a specific cohort of patients at increased risk for AAAs. Screening should be done only in patients who would be considered possible operative candidates, in whom intervention then could be considered.

We suggest consideration of screening for all men aged $65-80$ years and for women aged $65-80$ years with a history of smoking or cardiovascular disease. Screening can be considered in patients older than 80 years, depending on life expectancy and patient choice. In contrast to other recent guidelines, ${ }^{16}$ we believe the evidence to exclude women from AAA screening programs does not exist. Given real-world data suggesting the devastating natural history of AAA in women compared with men, and the sizeable proportion of women among patients who are treated for AAA, it is imperative that women not be excluded from screening programs.

Screening first-degree family members of patients with an AAA should be considered after the age of 55 . For those who have been found to have abdominal aortic ectasia with an initial aortic diameter of $2.5-3 \mathrm{~cm}$, repeat ultrasonography after 10 years should be considered depending on life expectancy and patient choice.

There exists a need for a national screening program in Canada, similar to those in other countries. With such a program identifying patients at high risk as per our recommendations, many deaths from a ruptured AAA event could be prevented. Further study into the cost-effectiveness of these recommendations will be required and is currently being considered by the CSVS.

Affiliations: From the William Osler Health System, Brampton, Ont. (Kapila); the University of Ottawa, Ottawa, Ont. (Jetty); the University of Toronto, Toronto, Ont. (Wooster); Trillium Health Partners, Mississauga, Ont. (Vucemilo); and Western University, London, Ont. (Dubois).

Competing interests: Doug Wooster received support for attending meetings and/or travel from the Vascular Surgery Academic Fund, University Health Network. He is cochair of the Quality Improvement and Research Committee, Society for Vascular Ultrasound, medical director of the Toronto West Vascular Lab, a peer reviewer for the College of Physicians and Surgeons of Ontario (CPSO) and a reviewer for the CPSO Independent Health Facilities program. No other competing interests were declared.

Contributors: All authors designed the study, and acquired and analyzed the data. V. Kapila, P. Jetty, D. Wooster and L. Dubois wrote the manuscript, which all authors critically revised. All authors gave final approval of the article to be published.

Content licence: This is an Open Access article distributed in accordance with the terms of the Creative Commons Attribution (CC BYNC-ND 4.0) licence, which permits use, distribution and reproduction in any medium, provided that the original publication is properly cited, the use is noncommercial (i.e., research or educational use), and no modifications or adaptations are made. See: https://creativecommons. org/licenses/by-nc-nd/4.0/.

\section{References}

1. Kuivaniemi H, Ryer EJ, Elmore JR, et al. Understanding the pathogenesis of abdominal aortic aneurysms. Expert Rev Cardiovasc Ther 2015;13:975-87.

2. Ashton HA, Buxton MJ, Day NE, et al. The Multicentre Aneurysm Screening Study (MASS) into the effect of abdominal aortic aneurysm screening on mortality in men: a randomised controlled trial. Lancet 2002;360:1531-9.

3. Moll FL, Powell JT, Fraedrich G, et al.; European Society for Vascular Surgery. Management of abdominal aortic aneurysms clinical practice guidelines of the European Society for Vascular Surgery. Eur 7 Vasc Endovasc Surg 2011;41(Suppl 1):S1-58.

4. Medical Advisory Secretariat. Ultrasound screening for abdominal aortic aneurysm: an evidence-based analysis. Ont Health Technol Assess Ser 2006;6:1-67.

5. Mastracci TM, Cinà CS; CSVS. Screening for abdominal aortic aneurysm in Canada: review and position statement of the Canadian Society for Vascular Surgery. 7 Vasc Surg 2007;45:1268-76.

6. Stather PW, Dattani N, Bown MJ, et al. International variations in AAA screening. Eur 7 Vasc Endovasc Surg 2013;45:231-4.

7. Lindholt JS, Sørensen J, Søgaard R, et al. Long-term benefit and cost-effectiveness analysis of screening for abdominal aortic aneurysms from a randomized controlled trial. Br 7 Surg 2010;97:826-34.

8. McCaul KA, Lawrence-Brown M, Dickinson JA, et al. Long-term outcomes of the Western Australian Trial of Screening for Abdominal Aortic Aneurysms: secondary analysis of a randomized clinical trial. FAMA Intern Med 2016;176:1761-7.

9. Thompson SG, Ashton HA, Gao L, et al.; Multicentre Aneurysm Screening Study (MASS) Group. Final follow-up of the Multicentre Aneurysm Screening Study (MASS) randomized trial of abdominal aortic aneurysm screening. Br F Surg 2012;99:1649-56.

10. Ashton HA, Gao L, Kim LG, et al. Fifteen-year follow-up of a randomized clinical trial of ultrasonographic screening for abdominal aortic aneurysms. Br F Surg 2007;94:696-701. 
11. Guirguis-Blake JMBT, Sun X, Senger CA, et al. Primary care screening for abdominal aortic aneurysm: an evidence update for the U.S. Preventive Services Task Force. Rockville (MD): Agency for Healthcare Research and Quality; 2014.

12. Chaikof EL, Dalman RL, Eskandari MK, et al. The Society for Vascular Surgery practice guidelines on the care of patients with an abdominal aortic aneurysm. 7 Vasc Surg 2018;67:2-77.e2.

13. Wanhainen A, Hultgren R, Linné A, et al. Outcome of the Swedish Nationwide Abdominal Aortic Aneurysm Screening Program. Circulation 2016;134:1141-8.

14. Jacomelli J, Summers L, Stevenson A, et al. Impact of the first 5 years of a national abdominal aortic aneurysm screening programme. $\mathrm{Br} \mathcal{F}$ Surg 2016;103:1125-31.

15. Scott RA, Bridgewater SG, Ashton HA. Randomized clinical trial of screening for abdominal aortic aneurysm in women. Br 7 Surg 2002; 89:283-5.

16. Canadian Task Force on Preventive Health Care. Recommendations on screening for abdominal aortic aneurysm in primary care. CMAf 2017;189:E1137-45.

17. Guyatt GH, Oxman AD, Vist GE, et al.; GRADE Working Group. GRADE: an emerging consensus on rating quality of evidence and strength of recommendations. BM7 2008;336:924-6.

18. Oliver-Williams C, Sweeting MJ, Turton G, et al. Lessons learned about prevalence and growth rates of abdominal aortic aneurysms from a 25 -year ultrasound population screening programme. $B r f$ Surg 2018;105:68-74.

19. Svensjö S, Björck M, Gurtelschmid M, et al. Low prevalence of abdominal aortic aneurysm among 65-year-old Swedish men indicates a change in the epidemiology of the disease. Circulation 2011;124:1118-23.

20. Svensjö S, Mani K, Björck M, et al. Screening for abdominal aortic aneurysm in 65-year-old men remains cost-effective with contemporary epidemiology and management. Eur 7 Vasc Endovasc Surg 2014;47:357-65.

21. Kung HC, Hoyert DL, Xu J, et al. Deaths: final data for 2005. Natl Vital Stat Rep 2008;56:1-120.

22. Mureebe L, Egorova N, Giacovelli JK, et al. National trends in the repair of ruptured abdominal aortic aneurysms. F Vasc Surg 2008;48:1101-7.

23. Brown LC, Powell JT. Risk factors for aneurysm rupture in patients kept under ultrasound surveillance. UK Small Aneurysm Trial Participants. Ann Surg 1999;230:289-96, discussion 96-7.

24. Skibba AA, Evans JR, Hopkins SP, et al. Reconsidering gender relative to risk of rupture in the contemporary management of abdominal aortic aneurysms. 7 Vasc Surg 2015;62:1429-36.

25. Sidloff DA, Saratzis A, Sweeting MJ, et al. Sex differences in mortality after abdominal aortic aneurysm repair in the UK. Br 7 Surg 2017;104:1656-64.

26. Dubois L, Novick TV, Harris JR, et al. Outcomes after endovascular abdominal aortic aneurysm repair are equivalent between genders despite anatomic differences in women. 7 Vasc Surg 2013;57:382-9. e1.

27. Nelson HD, Tyne K, Naik A, et al. Screening for breast cancer: an update for the U.S. Preventive Services Task Force. Ann Intern Med 2009;151:727-37, W237-42

28. Rembold CM. Number needed to screen: development of a statistic for disease screening. BMf 1998;317:307-12.

29. Norman PE, Jamrozik K, Lawrence-Brown MM, et al. Population based randomised controlled trial on impact of screening on mortality from abdominal aortic aneurysm. BM7 2004;329:1259.

30. Lederle FA, Larson JC, Margolis KL, et al.; Women's Health Initiative Cohort Study. Abdominal aortic aneurysm events in the Women's Health Initiative: cohort study. BMF 2008;337:a1724.

31. IMPROVE Trial Investigators. Comparative clinical effectiveness and cost effectiveness of endovascular strategy $\mathrm{v}$ open repair for ruptured abdominal aortic aneurysm: three year results of the IMPROVE randomised trial. BMf 2017;359:j4859.

32. Kansal V, Nagpal S, Jetty P. Editor's choice - Late open surgical conversion after endovascular abdominal aortic aneurysm repair. Eur 7 Vasc Endovasc Surg 2018;55:163-9.
33. Derubertis BG, Trocciola SM, Ryer EJ, et al. Abdominal aortic aneurysm in women: prevalence, risk factors, and implications for screening. 7 Vasc Surg 2007;46:630-5.

34. Glover MJ, Kim LG, Sweeting MJ, et al. Cost-effectiveness of the National Health Service Abdominal Aortic Aneurysm Screening Programme in England. Br 7 Surg 2014;101:976-82.

35. Lo RC, Bensley RP, Hamdan AD, et al. Gender differences in abdominal aortic aneurysm presentation, repair, and mortality in the Vascular Study Group of New England. 7 Vasc Surg 2013;57:1261-8, 1268.e1-5.

36. Deery SE, Soden PA, Zettervall SL, et al. Sex differences in mortality and morbidity following repair of intact abdominal aortic aneurysms. 7 Vasc Surg 2017;65:1006-13.

37. Makrygiannis G, Labalue P, Erpicum M, et al. Extending abdominal aortic aneurysm detection to older age groups: preliminary results from the Liège Screening Programme. Ann Vasc Surg 2016;36:55-63.

38. Prenner SB, Turnbull IC, Malik R, et al. Outcome of elective endovascular abdominal aortic aneurysm repair in octogenarians and nonagenarians. 7 Vasc Surg 2010;51:1354-9.

39. Han Y, Zhang S, Zhang J, et al. Outcomes of endovascular abdominal aortic aneurysm repair in octogenarians: meta-analysis and systematic review. Eur 7 Vasc Endovasc Surg 2017;54:454-63.

40. Howard DP, Banerjee A, Fairhead JF, et al.; Oxford Vascular Study. Age-specific incidence, risk factors and outcome of acute abdominal aortic aneurysms in a defined population. Br 7 Surg 2015;102:907-15.

41. Kuivaniemi H, Shibamura H, Arthur C, et al. Familial abdominal aortic aneurysms: collection of 233 multiplex families. 7 Vasc Surg 2003;37:340-5.

42. Sandford RM, Brown MJ, London NJ et al. The genetic basis of abdominal aortic aneurysms: a review. Eur 7 Vasc Endovasc Surg 2007; 33:381-90.

43. Darling RC 3rd, Brewster DC, Darling RC, et al. Are familial abdominal aortic aneurysms different? 7 Vasc Surg 1989;10:39-43.

44. Baird PA, Sadovnick AD, Yee IM, et al. Sibling risks of abdominal aortic aneurysm. Lancet 1995;346:601-4.

45. Linné A, Lindström D, Hultgren R. High prevalence of abdominal aortic aneurysms in brothers and sisters of patients despite a low prevalence in the population. F Vasc Surg 2012;56:305-10.

46. Ogata T, MacKean GL, Cole CW, et al. The lifetime prevalence of abdominal aortic aneurysms among siblings of aneurysm patients is eightfold higher than among siblings of spouses: an analysis of 187 aneurysm families in Nova Scotia, Canada. 7 Vasc Surg 2005;42:891-7.

47. Sakalihasan N, Defraigne JO, Kerstenne MA, et al. Family members of patients with abdominal aortic aneurysms are at increased risk for aneurysms: analysis of 618 probands and their families from the Liege AAA Family Study. Ann Vasc Surg 2014;28:787-97.

48. Linné A, Forsberg J, Lindström D et al. Age at detection of abdominal aortic aneurysms in siblings of patients with abdominal aortic aneurysms. 7 Vasc Surg 2016;63:883-7.

49. Webster MW, St Jean PL, Steed DL, et al. Abdominal aortic aneurysm: results of a family study. 7 Vasc Surg1991;13:366-72.

50. Verloes A, Sakalihasan N, Koulischer L, et al. Aneurysms of the abdominal aorta: familial and genetic aspects in three hundred thirteen pedigrees. 7 Vasc Surg 1995;21:646-55.

51. Frydman G, Walker PJ, Summers K, et al. The value of screening in siblings of patients with abdominal aortic aneurysm. Eur 7 Vasc Endovasc Surg 2003;26:396-400.

52. Linné A, Forsberg J, Leander K, et al. Screening of siblings to patients with abdominal aortic aneurysms in Sweden. Scand Cardiovasc 7 2017;51:167-71.

53. Hultgren R, Linné A, Svensjö S. Cost-effectiveness of targeted screening for abdominal aortic aneurysm in siblings. Br F Surg 2019; 106:206-16.

54. Söderberg P, Wanhainen A, Svensjö S. Five year natural history of screening detected sub-aneurysms and abdominal aortic aneurysms in 70 year old women and systematic review of repair rate in women. Eur 7 Vasc Endovasc Surg 2017;53:802-9. 